Introduction BASHH recommends Lymphogranuloma venereum (LGV) testing of Chlamydia trachomatis (CT)-positive specimens from men who have sex with men (MSM) presenting with proctitis, and all rectal CT from HIV-positive MSM. Until recently in England, LGV testing was only available as a referred test at the Sexually Transmitted Bacteria Reference Unit (STBRU). In July 2016 we implemented a validated inhouse version of the STBRU LGV PCR on all CT-positive specimens from MSM, regardless of symptoms or HIV status. We assessed the time from specimen collection to result (turnaround time, TRT) and defined clinical features of LGV cases. Methods From July 2016 to March 2017 we reviewed all positive LGV tests, recording patients' demographics, HIV status, chemsex behaviour, presence of symptoms and LGV result TRT.

Results We conducted 587 LGV tests on CT-positive specimens from MSM, of which $50(8.5 \%)$ were positive. Median age of LGV cases was 38 (range 23 to 65), 28 (56\%) were Caucasian, $38(76 \%)$ were HIV positive and chemsex behaviour was reported by $20(40 \%) ; 12$ patients $(24 \%)$ had a past history of LGV. Nine (18\%) cases were asymptomatic and three of these were HIV-negative MSM. The mean TRT was 12 days (range 8 to 20); compared with 35 days (range 15 to 118) in the six months prior to in-house testing.

Discussion LGV continues to occur mainly in HIV-positive MSM as symptomatic proctitis. Testing all CT-positive MSM increased detection of LGV compared with following BASHH guidelines, and in-house testing reduced TRT significantly.

\section{I WAS STRUGGLING TO FEEL INTIMATE, THE DRUGS JUST HELPED'. CHEMSEX AND HIV-RISK AMONG MEN WHO HAVE SEX WITH MEN (MSM) IN THE UK: SYNDEMICS OF STIGMA, MINORITY-STRESS, MALADAPTIVE COPING AND RISK ENVIRONMENTS}

${ }^{1}$ Alex Pollard*, 2,3 Tom Nadarzynski, ${ }^{1}$ Carrie Llewellyn. 'Brighton and Sussex Medical School, Brighton, East Sussex, UK; ${ }^{2}$ Solent NHS Trust, Southampton, Hampshire, UK; ${ }^{3}$ University of Southampton, Southampton, Hampshire, UK

\subsection{6/sextrans-2017-053232.13}

Introduction There has been a steep rise in the use of drugs during sex by some men who have sex with men (MSM), with associated increases in sexual risk for HIV and other STIs. This 'Chemsex' has been described, but there is a lack of theoretical perspectives applied to this particular phenomenon.

We aimed to assess participants' reasoning and conceptualisation of Chemsex and situate this within theoretical frameworks.

Methods This study presents data from telephone interviews with $15 \mathrm{MSM}$ attending sexual health clinics following a risk of HIV and accessing post-exposure prophylaxis (PEP). Interviews were conducted as part of a larger interventional study, which used an adapted version of Motivational Interviewing to explore risk behaviour and support change. We used Framework analysis on interview transcripts in order to understand participants' perspectives on the use of chemsex.

Results Participants conceptualised their chemsex and HIV risks in their psycho-social context, highlighting the influence of the psycho-socio-cultural challenges of homophobic marginalisation and the 'gay scene' on their behaviour. Narratives of loneliness and difficulties in forming satisfying social and sexual relationships were repeatedly identified.
Discussion Multiple influences of stigma, minority stress and maladaptive coping (including drug-use) are seen to contribute to syndemic 'risk-environments' in which chemsex and risk behaviours are played out. Interventions to address the harms of chemsex must recognise the complex psychosocial context of risk, and shift the responsibility for change from vulnerable individuals to a shared responsibility distributed across social, political and institutional contexts.

\section{CHEMSEX, CONSENT AND THE RISE IN SEXUAL ASSAULT}

Chris Ward*, Orla McQuillan, Rebecca Evans. The Northern contraception, sexual health and HIV service, Manchester, UK

\subsection{6/sextrans-2017-053232.14}

Introduction Within the chemsex population reports of sexual assault, non-consensual sex and coercion are rising. We looked at consent among our chemsex clinic users.

Methods Retrospective data review of patients from April 2015 to March 2017. Data was collected on sexual assault, coercion, exploitation, risk taking, sexually transmitted infections and drug use.

Results 72 men were seen with a median age of 32.41 (56.9\%) were HIV positive, and 11 (15.3\%) had Hepatitis C. $53(73.6 \%)$ patients used Mephedrone, 40 (55.6\%) GHB and $22(30.6 \%)$ Crystal Meth. $13(18.1 \%)$ patients reported selfharm. In total $23(31.9 \%)$ patients reported non-consensual sex. A minority 5/30 (16.7\%) were identified from April 2015 to Jan 2016 when using the terminology 'forced into sex'. After realising that addressing consent is more complex in this cohort, we prioritised consent discussions around unwanted sexual attention and from Jan 2016 to March 2017 18/42 (42.9\%) reported non-consensual sex (Table 1).

\begin{tabular}{ll} 
Abstract 014 Table 1 & Chemsex \\
\hline Assault/coercion & $\mathrm{N} / 42(\%)$ \\
\hline Non-consensual sex & $18(42.9 \%)$ \\
Reported as sexual assault & $6(14.3 \%)$ \\
Coercive sex & $4(9.5 \%)$ \\
Sex while unconscious & $3(7.1 \%)$ \\
Assaulted > once & $2(4.8 \%)$ \\
Allegations of organised assault & $2(4.8 \%)$ \\
Injected/filmed while unconscious & $1(2.4 \%)$ \\
\hline
\end{tabular}

Discussion Our data shows rates of non-consensual sex among chemsex users of up to $42.9 \%$. There is a lack of patient understanding around what sexual assault and consent are and exploring this in a sensitive manner is paramount. Sexual assault discussions must be reviewed in both standard sexual health and chemsex clinics.

\section{A SERVICE EVALUATION COMPARING HOME-BASED TESTING TO CLINIC-BASED TESTING FOR CHLAMYDIA AND GONORRHOEA IN BIRMINGHAM AND SOLIHULL}

Prita Banerjee*, Nicola Thorley, Keith Raddliffe. University Hospital Birmingham, Birmingham, UK

10.1136/sextrans-2017-053232.15 